\title{
Explosive wave propagation in the presence of antiseismic protective curtain
}

\author{
Viktor Kravets ${ }^{1 *}$, Nataliia Remez $^{1}$, Andrii Kovtun ${ }^{1}$, and Azer Shukiurov ${ }^{1}$ \\ ${ }^{1}$ National Technical University of Ukraine "Igor Sikorsky Kyiv Polytechnic Institute", Department of \\ Geobuilding and Mining Technologies, 37 Peremohy Ave., 30056 Kyiv, Ukraine
}

\begin{abstract}
The objective of this work is to study theoretically the stress wave formation in the borehole-to-borehole space and on the boundary with the borehole contour set by creating a directed fracture system in it. The features of an interaction of the weak stress wave at a large-scale blasting in the short-delay blast mode (SDB) with the massif and the protective contour set of boreholes, are considered. It is noted the effect of the contour set parameters on wave processes. The constructive solutions are proposed in order to reduce the dynamic loadings in the peripheral rock massif. The proposals consist in applying a combined construction of garland charges to form the seismo protective structure that consists of a contour fracture and one-sided disrupted zone within the boundaries of the blasted block.
\end{abstract}

\section{Introduction}

The development of a seismically secure switching circuit for detonating system of largescale blasting involves the primary blast of a contour set of boreholes [1], which reduces the loading from the last-line blast on the peripheral rock massif, comes out from the simplified representation about charge groups interaction. In this case, three consecutive mechanisms of interaction are considered - at the level of interference of the stress waves, at the level of the fracture system development and at the level of contact between the rocks blocks, bounded by adjacent groups. The first level provides the preliminary disturbance of microstructural bonds in the massif being destroyed, the development of microdefects, and the creation of a stress state in the massif. The following two levels practically solve the problem of separating the moving massif into standard sized parts. However, under real conditions of large-scale blasting, the processes of charge interaction and the resulting dynamic phenomena [2] become more complicated, acquiring additional features that should be studied.

\section{Prerequisites for setting the research tasks}

When implementing a large-scale blasting in the SDB mode, a relatively long timeextended oscillation field, formed in three stages, is actually created in the rock massif.

\footnotetext{
*Corresponding author: kravets@geobud.kiev.ua
} 
These stages involve the blast of borehole charges in a certain sequence.

The first stage is realized during hundreds of microseconds in the process of sequential dynamic loading of the massif during the detonation decomposition of a separate elongated charge in the group and the radiation from shock wave at its contact with the rock massif. Taking into account that the propagation velocity of the detonation wave in the charge is of $4-5$ thousand $\mathrm{m} / \mathrm{s}$ for modern explosives, at a charge length of $10 \mathrm{~m}$, its detonation decomposition will be about 500 microseconds. With the parameters of the system of borehole charges $5 \times 5 \mathrm{~m}$, during this time, the stress wave front at the level of the intermediate detonator (gunner) activation at its velocity in the hard rock also about 5 thousand $\mathrm{m} / \mathrm{s}$, will cross the 2 next groups of charges in the SDB scheme, which should be blasted much later - in tens of milliseconds. In this case, the stress wave front is located at an angle of $45^{\circ}$ to the horizontal. Accordingly, there are changes in geometry of the rock masses displacement and interaction, driven by the charges in the set, as well as adjacent groups.

Thus, in the interval between two adjacent groups of charges due to non-simultaneous occurrence of the stress wave, the time difference in dynamic "pre-destruction" can be about 500 microseconds, and the time of the first arrival of stress front on an adjacent group will be 250 microseconds.

The second mode is related to the sequence of blasting the borehole charges in a group. Based on the features of the charge system assembly, the first and last charge in the group only theoretically should be blasted simultaneously. In fact, these charges are activated in sequence or along a common segment of the detonating cord, as well as in case of application of individual segments of the detonating waveguide of any modern initiation system of the "NONEL" type. In the latter variant, each subsequent charge in the group at a waveguide detonation velocity of 2 thousand $\mathrm{m} / \mathrm{s}$ will be activated through 2.5 milliseconds relative to the previous one. Together, the whole group of 5 charges will be activated in 10 milliseconds.

The third mode assumes a consistent blasting of the charge groups with a deceleration within a few tens of milliseconds (from 20 to $70-100 \mathrm{~ms}$ ).

\section{Material and research results}

The processes of blasting in mining and construction are accompanied by the propagation of stress waves outside and within the boundary of the massif being destroyed, which along with the massif disruption to the specified granulometric composition, have a harmful effect on the surrounding buildings, mine workings and the massif itself in the form of elasticplastic (residual) and elastic deformation [3]. With account of the danger of seismic phenomena within the boundary of the bench which should be destroyed, it is considered appropriate, if possible, to protect the massif outside the blast block from damage, which in the future will lead to a weakening of the massif adjacent to disintegration of rock. These phenomena will either be a source of increased oversized output, or will need to increase the explosives consumption. Consequently, a decrease in the seismic effect outside the massif being blasted will reduce the explosive materials (EM) consumption.

In the specific conditions of production, the question arises of developing an integrated approach to managing the explosion energy - increasing the fraction of energy consumed for destruction, and reducing the seismic waves energy. One of such effective method of influencing the result of large-scale blasting is shielding [1].

The essence of the shielding method is in the formation on the boundary of the block, which should be destroyed, of the contour set of boreholes designed in two variants - either with the formation of a continuous mono gapping in the plane of contour charges system, or a plane zone of intense fracturing. The contour set being a curtain on the boundary of the 
surrounding massif from seismic oscillations, which reflects, scatters and refracts the waves generated by the blast. Outside the shield, the refracted wave carries only a part of the energy into the medium. It is important to note that the energy of the reflected wave, returning to the destroyed massif, increases the destructive effect of the blast.

Physical phenomena that contribute to the shielding process have not been studied sufficiently, therefore, it is proposed to apply the shields in a complex, that is, to apply the common and new types of shields with altered geometric, kinematic and dynamic parameters in order to increase the effectiveness of blasting operations. Solving problems on optimal parameters determination of such shields will ultimately increase the blast destruction effectiveness and reduce the seismic effect in the protected zone.

Such shielding consists in creating on the boundary of a blasted block, a zone of broken rock with a correspondingly reduced bandwidth throughput for elastic waves of sufficiently high intensity. In general, the effectiveness of destruction and the degree of shielding is estimated by the ratio of mass velocities in the massif with and without the shield at the equal distances from the charge.

To assess the capabilities of such a shield, let us consider in a simplified form the interaction of two adjacent boreholes filled with a gas-generating emulsion substance, wherein the first charge performs the role of active, that is detonating charge, the second performs the passive role.

In the first place, the patterns are investigated of stress wave propagation into a rock massif if to blast the cylindrical explosive charge, as well as its interaction with the material filling the adjacent borehole.

The movement of the detonation products, the mine rock and explosive materials are studied within the framework of continuum mechanics [4]. This motion is described by the laws of momentum and mass conservation. For the case of axial symmetry, they have the form [5]:

$$
\begin{gathered}
\frac{\partial \sigma_{r r}}{\partial z}+\frac{\partial \tau_{r z}}{\partial r}+\frac{\tau_{r z}}{r}=\rho \frac{d u}{d t}, u=\frac{d z}{d t} ; \\
\frac{\partial \tau_{r z}}{\partial z}+\frac{\partial \sigma_{z z}}{\partial r}+\frac{\sigma_{z z}-\sigma_{\theta \theta}}{r}=\rho \frac{d w}{d t}, w=\frac{d r}{d t} ; \\
\frac{1}{V} \frac{d V}{d t}=\frac{\partial u}{\partial z}+\frac{\partial w}{\partial r}+\frac{w}{r} ; \\
\sigma_{z z}=S_{z z}-P, \sigma_{r r}=S_{r r}-P, \sigma_{\theta \theta}=S_{\theta \theta}-P ; \\
P=\frac{1}{3}\left(\sigma_{r r}+\sigma_{\theta \theta}+\sigma_{z z}\right) ; V=\frac{\rho_{0}}{\rho},
\end{gathered}
$$

where $z, r$ are the coordinates; $t$ is the time; $\sigma_{r r}, \sigma_{\theta \theta}, \sigma_{z z}$ are the normal stresses; $\tau_{z r}$ is tangential stress; $S_{z z}, S_{r r}, S_{\theta \theta}$ are the components of the stress tensor deviator; $P$ is the average hydrostatic pressure; $\rho$ is the density; $u, w$ are velocity components, $V$ is the specific volume.

The extension of the detonation products (DP) occurs by the binomial isentrope, the equation of which is proposed in [6]:

$$
P^{n}=A \cdot \rho^{n}+B \rho^{\gamma+1},
$$

where $A, B, n, \gamma$ are the constants for this explosive material. 
For mine rocks, the ratios between stresses and deformations are written on the basis of the differential theory of plasticity [7]. The values of the deformation tensor components are represented as the sum of the elastic and plastic deformations:

$$
\varepsilon_{r r}=\varepsilon_{r r}^{\prime}+\varepsilon_{r r}^{\prime \prime}, \varepsilon_{z z}=\varepsilon_{z z}^{\prime}+\varepsilon_{z z}^{\prime \prime}, \varepsilon_{r z}=\varepsilon_{r z}^{\prime}+\varepsilon_{r z}^{\prime \prime}
$$

The connection between the stress and elastic deformations is established by the formulas of the generalized Hooke's law for isotropic material:

$$
\sigma_{r r}=\frac{E}{1-v^{2}}\left(\varepsilon_{r r}^{\prime}+\varepsilon_{z z}^{\prime \prime}\right), \quad \sigma_{z z}=\frac{E}{1-v^{2}}\left(\varepsilon_{z z}^{\prime}+v \varepsilon_{r r}^{\prime}\right), \quad \tau_{r z}=G \varepsilon_{r z}^{\prime},
$$

where $E, G, v$ are the physical and mechanical characteristics of the mine rock.

In the case of plastic deformations, the ratio between the corresponding deformations and stresses is written as:

$$
d \varepsilon_{r r}^{\prime \prime}=\lambda S_{r r}, \quad d \varepsilon_{z z}^{\prime \prime}=\lambda S_{z z}, d \varepsilon_{r z}^{\prime \prime}=\lambda S_{r z}
$$

when using the plasticity condition with linear kinematic displacement:

$$
S_{r r}^{2}+S_{z z}^{2}+2 S_{r z}^{2} \leq \frac{2}{3} \sigma_{T}^{2}
$$

where $\lambda$ is the coefficient of displacement; $\sigma_{T}$ is the ultimate strength.

The emulsion explosive materials (EEM) of a cylindrical charge is modeled by twocomponent nonlinear-elastic medium, which consists of air and liquid components [8]. It is believed that under the action of dynamic loading, each component has the same pressure and moves at the same velocity. At atmospheric pressure, the density of EM is determined by the formula:

$$
\rho_{0}=\alpha_{1} \rho_{10}+\alpha_{2} \rho_{20}, \alpha_{1}+\alpha_{2}=1
$$

where $a_{1}, a_{2}$ and $\rho_{10}, \rho_{20}$ is the volumetric content of components and their density.

The equation of volumetric compression of nonlinear-elastic two-component medium has the form:

$$
\frac{\rho_{0}}{\rho}=\sum_{i=1}^{2} \alpha_{i}\left[\frac{\gamma_{i}\left(P-P_{0}\right)}{\rho_{i 0} c^{2}{ }_{i 0}}+1\right]^{-1 / \gamma_{i}},
$$

where $c_{i 0}, \gamma_{i}$ is the sound velocuty and the index of isentrope of the $i$-th component.

The initial conditions of the problem are the following:

$$
\begin{aligned}
& u=0, w=0, P=P_{n}, \rho=\rho_{n} \text { at } z<l, r<r_{0} ; \\
& u=0, w=0, P=P_{0}, \rho=\rho_{0} \text { at } z>l, r>r_{0} .
\end{aligned}
$$

The boundary conditions are:

$$
\begin{aligned}
& u_{D P}=u_{R M}, P_{D P}=P_{R M}, \rho_{D P} \neq \rho_{R M} \text { at } z=l ; \\
& w_{D P}=w_{R M}, P_{D P}=P_{R M}, \rho_{D P} \neq \rho_{R M} \text { at } r=r .
\end{aligned}
$$

The $D P$ and $R M$ are the indices of the detonation products and rock mass, respectively.

To solve the problem, the finite difference method is chosen in a moving Lagrangian coordinate system with a moving grid, which is automatically extended at the end of each computational cycle. The finite-difference scheme of the 'cross' type of the second order of 
accuracy [5] was used. The algorithm and the program for the PC were developed. In the numerical solution of the problem, a blast of EEM charge in granite was considered.

The detonation characteristics of the EEM are the following: $P_{n}=3.9 \cdot 10^{9} \mathrm{~Pa}$, $\rho_{n}=1200 \mathrm{~kg} / \mathrm{m}^{3}, D=5000 \mathrm{~m} / \mathrm{c}, n=1.4$.

The parameters for the state equation of the DP (6) are as follow:

$$
A=56.68, B=0.68 \cdot 10^{5}, n=2.482, \gamma_{0}=0.242 .
$$

The cylindrical charge has a diameter of $0.2 \mathrm{~m}$ and a length of $15 \mathrm{~m}$.

The physical and mechanical parameters of the mine rock are as follows:

$$
\rho_{0}=2650 \mathrm{~kg} / \mathrm{m}, c_{p}=4170 \mathrm{~m} / \mathrm{c}, c_{s}=2800 \mathrm{~m} / \mathrm{c}, E=4.6 \cdot 10^{10} \mathrm{~Pa}, v=0.09 .
$$

Fig. 1 shows the theoretical dependence of the initial pressure on the time in the mine rock at the boundary with the detonation products during the blast of the EEM cylindrical charge. As shown in Fig. 1, the pulse has a characteristic shape for the shock wave. According to the theory, the maximum pressure in the shock wave is achieved almost instantaneously (in fact, about 200 microseconds), but the time of the stress decrease to 0 reaches 100 milliseconds.

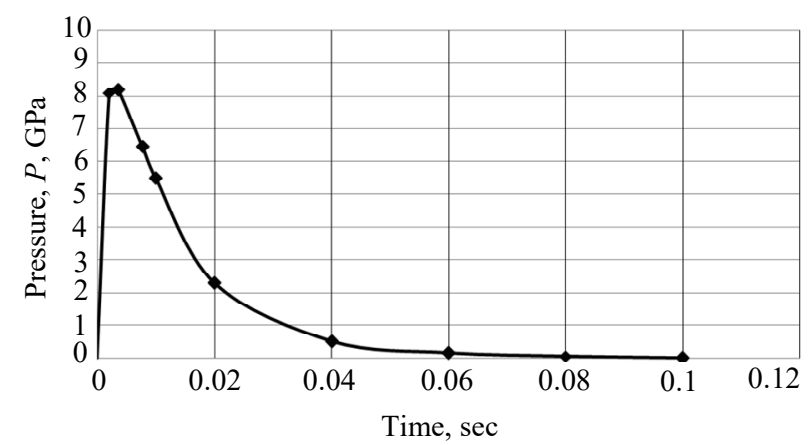

Fig.1 The curve of pressure in the mine rock at the boundary with the detonation products of the EEM cylindrical charge.

Since the energy of the explosive pulse is commensurate with the area of the curve shown in Fig. 1, the pulse existence time is extremely important. A known means for reducing the maximum pressure level in the initial pulse with the simultaneous lengthening of the pulse existence time while maintaining the total area of the pulse is the use of air gaps in the charge, that is, the dispersion of the elongated charge. This method leads to a decrease in $\mathrm{P}_{\mathrm{MAX}}$, reducing the size of the zone of rock overmilling and, consequently, the energy loss in the near area of the blast effect, and at the same time increases the pulse existence time. Due to energy losses in the zone of rock overmilling, or in the plastic deformations zone, the shock wave quickly transforms into a stress wave.

The further development of the process is shown in Fig. 2, where curve 1 corresponds to the dependence of the maximum pressure distribution (MPa) in the incident stress wave, which propagates into the borehole-to-borehole space $a=5 \mathrm{~m}$ in the mine rock, the curve 2 - in the reflected stress wave. The reflection phenomenon is a sign of the effectiveness of the obstacle in the path of the incident stress wave movement.

From Fig. 2 it is evident that in the borehole-to-borehole space at a distance of $25-35$ radii of the active (detonating) charge, a significant jump in the pressure is fixed compared to the background one. The greatest pressure in the reflected wave is observed in the mine 
rock at a distance of 30 charge radii $(3 \mathrm{~m})$ from the active charge, accounting for more than $7 \mathrm{MPa}$. At the boundary with the adjacent borehole $\left(R_{0}=45\right)$, the value of the average hydrostatic pressure reaches 3.8 MPa (Fig. 2).

Since the passive borehole in the task plays the role of the shielding cavity, it should be filled with an effective absorbing material. If such a shield has to exist for a certain time, it should simultaneously keep the borehole walls from being destructed under the influence of dynamic loadings. In the task we use the condition of filling the borehole with gasgenerating emulsion material in two variants (Fig. 3).

The dependence of the density on the coordinate in the passive charge under the action of the stress wave of the active charge has a nonlinear character, since the emulsion is deformed in accordance with the law of the nonlinear-elastic medium. As it follows from the calculations (Fig. 3), this compliant material with an initial density of $1200 \mathrm{~kg} / \mathrm{m}^{3}$ under the action of $3.8-3.5 \mathrm{MPa}$ pressure acquires a new density. In the first variant, the emulsion density increases on the front wall of the borehole from 1200 to $1478.5 \mathrm{~kg} / \mathrm{m}^{3}$, and when the wave passes through the borehole, it decreases to $1476 \mathrm{~kg} / \mathrm{m}^{3}$ on the opposite wall. In practice, one can consider such a change insignificant. This means that since in the first variant of the problem the emulsion is only $2 \%$ saturated with gas bubbles, its role as a means of absorbing the wave energy is insignificant.

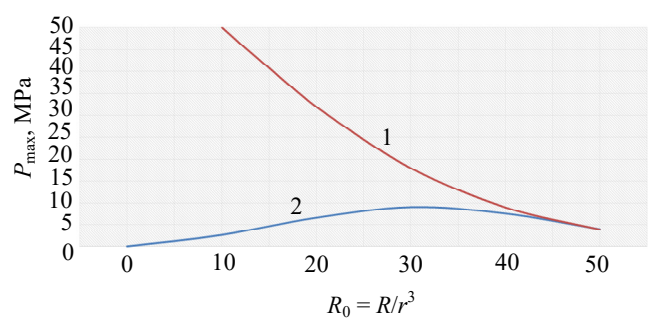

Fig. 2. The distribution of the maximum pressure $P_{\max }$ in incident (1) and reflected (2) stress waves between adjacent boreholes.

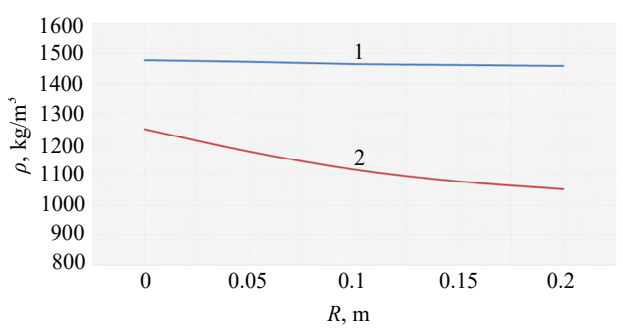

Fig. 3. The density distribution of the porous filler under the action of a stress wave in the section of the contour borehole with an emulsion, gasgenerated with sensitizer in an amount: $1-2 \% ; 2-15 \%$.

In the second variant of the problem, an emulsion with a density of $800 \mathrm{~kg} / \mathrm{m}^{3}$ has been used as absorbing material. Based on the character of curve 2 in Fig. 3, it follows that by virtue of the greater absorption capacity of the shield material, thus, the dissipation efficiency of the stress wave energy also increases significantly. With a significant compaction of the emulsion material (up to $1250 \mathrm{~kg} / \mathrm{m}^{3}$ ) on the front wall of the borehole, its density on the opposite wall is much less and is $1050 \mathrm{~kg} / \mathrm{m}^{3}$. Such a decrease in density within the borehole diameter indicates a significant increase in the effectiveness of the shield material.

Consequently, the shield effectiveness depends to a large extent on the filler material, as evidenced by graphs 1 and 2 in Fig. 3. However, the filtering performance of the protective structure relative to the stress wave is determined by its size in the direction of the wave motion, which should be commensurate with the wavelength. It is known [9] that when the relative density of the shield material is 0.9 , the relative wave resistance is 0.63 , whereas at a relative density of the shield material 0.6 this value is reduced to 0.12 , that is, almost by 5 times. However, a change in the ratio of the shield width to the wavelength from 0.05 to 0.8 , that is, by 16 times, changes the ratio of energy in the refracted and incident waves from 0.65 to 0.33 , that is, only twice. So, if to consider the effect of these factors separately, the construction of a protective shield should begin with the selection of the filler material, 
and then to project the shield size.

Traditionally in mining, the contour set is arranged in such a way that the peripheral rock massif has got a minimum of damage. For this purpose, the diameter decreases of that boreholes, in which the garland charges of economical action are located, which are intended to create only fracture between the boreholes in the plane of the shield. Such a technique limits maximally the possibility of applying the main factor - shield density due to its inefficient width.

Based on the above, there is another technical solution. A variant of the complex method is possible, when the boreholes of increased diameter are drilled in the contour set to fill with the material absorbing the wave energy. To enhance the absorption capacity of the shield, the rock massif surrounding the borehole in the area wrapped up to the incident wave should be pre-saturated with a system of radial and slabbing fractures. In this case, the peripheral rock massif is minimally damaged.

To construct a contour shield, it is possible to use the properties of both factors in the complex by applying the garland charges of directional action. The peculiarities of the method is that the construction of such charges and the parameters of their location are based on the applying of systems of concentrated charges with a special shape, including the cumulative concavity. These charges are manufactured mainly at the blast site.

\section{Conclusions}

The analysis of the stress wave transformation in the conditions of shielding blast of the rock massif blocks by the system of borehole charges indicates the existence of a significant number of physical and technological factors determining the effectiveness of the contour method for protecting the rock massif adjacent to the place of large-scale blasting.

Theoretical studies and existing experience allow to develop and apply a new solution for the creation of a protective plane zone on the boundary of the large-scale blasting place with the adjacent rock massif. It is considered expedient to use the possibility of controlled formation of a protective fractured zone using the features of the mechanical effect development if to blast the concentrated charges with conic shape, which are manufactured at the site of blast operations.

Authors express their gratitude to the head of the Underground Mining Department at the National Mining University for help and consultations during the work.

\section{References}

1. Kravets, V.H., Korobiychuk, V.V., \&. Boiko, V.V. (2015). Fizychni protsesy prykladnoi heodynamiky vybukhu. Zhytomyr: Zhytomyrskyi derzhavnyi tekhnichnyi universytet.

2. Khomenko, O.Ye., \& Maltsev, D.V. (2005). K obosnovaniyu ratsional'noy tekhnologii burovzryvnykh rabot v usloviyakh shakht GP "VostGOK". Naukovyi Visnyk Natsionalnoho Hirnychoho Universytetu, (10), 3-6.

3. Bondarenko, V. Kovalevs'ka, I., \& Cherednychenko, Y. (2010). Substantiation of design and installation technology of tubular rock bolts by explosive method. New Techniques and Technologies in Mining, 9-14. https://doi.org/10.1201/b11329-3

4. Kovalevs'ka, I., Symanovych, G., \& Fomychov, V. (2013). Research of stress-strain state of cracked coal-containing massif near-the-working area using finite elements technique. Annual ScientificTechnical Collection - Mining of Mineral Deposits 2013, 159-163. https://doi.org/10.1201/b16354$\underline{28}$ 
5. Luchko, I.A., Plaksiy, V.A., \& Remez, N.S. (1989). Mekhanicheskiy effekt vzryva v gruntakh. Kyiv: Naukova dumka.

6. Fizika vzryva. (2002). Pod redaktsiyey L. Orlenko. Moskva: Fizmatlit.

7. Bondarenko, V., Kovalevs'ka, I., \& Fomychov, V. (2012). Features of carrying out experiment using finite-element methodat multivariate calculation of mine massif - combined support system. Geomechanical Processes During Underground Mining, 7-13. https://doi.org/10.1201/b13157-3

8. Lyakhov, G.M. (1982). Volny v gruntakh i poristykh mnogokomponentnykh sredakh. Moskva: Nauka.

9. Vovk, O.O. (mol.), Kravets, V.H., Isaienko, V.M., Vovk, O.O., \& Remez, N.S. (2014). Vplyv tekhnohennykh dynamichnykh protsesiv na stan pryrodnykh $i$ inzhenernykh obiektiv. Kyiv: Vydavnytstvo Natsionalnoho Pedahohichnoho Universytetu im. M.P. Drahomanova. 